\title{
A compact and customizable operation frequency filter for broadband applications
}

\author{
Yu Guo ${ }^{1,4}$, Shanwen $\mathrm{Hu}^{2}$, Xiaozhou Liu ${ }^{1 \mathrm{a})}$, Haodong $\mathrm{Wu}^{1 \mathrm{~b})}$, \\ Guojun Wang ${ }^{3}$, and Guann-Pyng $\mathbf{L i}^{4}$ \\ ${ }^{1}$ Key Laboratory of Modern Acoustics, Institute of Acoustics and School of Physics, \\ Nanjing University, NO. 22, Hankou Road, Nanjing, Jiangsu 210093, \\ People's Republic of China \\ ${ }^{2}$ Nanjing University of Posts and Telecommunications, \\ NO. 66, Xinmofan Road, Nanjing, Jiangsu 210003, People's Republic of China \\ ${ }^{3}$ School of Management and Engineering, Nanjing University, \\ NO. 22, Hankou Road, Nanjing, Jiangsu 210093, People's Republic of China \\ ${ }^{4}$ Department of Electrical Engineering and Computer Science, \\ University of California, Irvine, Irvine, CA 92697, USA \\ a)xzliu@nju.edu.cn \\ b)haodongwu@163.com
}

\begin{abstract}
A novel compact customizable EBG (Electromagnetic Bandgap) filter operating from 2.6 to $10.0 \mathrm{GHz}$ is proposed. The EBG filter is manufactured by a 2-layer PCB (Printed Circuit Board) process in RO4350 substrate with surface mounted lumped capacitors. A specific working frequency of the filter is selected on demand by adjusting the value of the capacitors, while the filter's performance is not compromised. The experimental results show a filter with size of $2.50 \times 3.00 \times 1.00 \mathrm{~mm}^{3}$ in PCB achieving performance of insertion loss of $3.34 \mathrm{~dB}, 1.19 \mathrm{~dB}$ and $1.20 \mathrm{~dB}$ at $2.60 \mathrm{GHz}, 7.71 \mathrm{GHz}$ and $10.0 \mathrm{GHz}$ with the capacitor of $5.1 \mathrm{pF}, 0.5 \mathrm{pF}$ and $0.3 \mathrm{pF}$ respectively.
\end{abstract}

Keywords: customizable, electromagnetic bandgap, filter, PCB

Classification: Microwave and millimeter wave devices, circuits, and systems

\section{References}

[1] L. Kurra, M. P. Abegaonkar, A. Basu and S. K. Koul: IEEE IMaRC (2013) 1. DOI:10.1109/IMaRC.2013.6777732

[2] Y. Deng and K. Wu: EuMC (2013) 1027.

[3] X. Liu, L. P. B. Katehi, W. J. Chappell and D. Peroulis: J. Microelectromech. Syst. 19 (2010) 774. DOI:10.1109/JMEMS.2010.2055544

[4] S. Moon, H. H. Sigmarsson, H. Joshi and W. J. Chappell: IEEE Microw. Wireless Compon. Lett. 20 (2010) 450. DOI:10.1109/LMWC.2010.2050680

[5] M. S. Arif and D. Peroulis: IEEE IMS (2012) 1. DOI:10.1109/MWSYM.2012. 6259684

[6] X. Gong, W. J. Chappell and L. P. B. Katehi: IEEE IMS 2 (2002) 1091. DOI:10.1109/MWSYM.2002.1011832 
[7] X. Gong, B. Liu, L. P. B. Katehi and W. J. Chappell: IEEE IMS 2 (2004) 425. DOI:10.1109/MWSYM.2004.1336000

[8] W. J. Chappell, M. P. Little and L. P. B. Katehi: IEEE IMS 3 (2000) 1437. DOI:10.1109/MWSYM.2000.862244

[9] W. J. Chappell, X. Gong and L. P. B. Katehi: IEEE IMS 3 (2003) 1611. DOI:10.1109/MWSYM.2003.1210446

[10] W. H. She, X. Gong and W. J. Chappell: IEEE APSURSI 2 (2004) 1419. DOI:10.1109/APS.2004.1330453

[11] W. J. Chappell, M. P. Little and L. P. B. Katehi: IEEE Microw. Wireless Compon. Lett. 11 (2001) 246. DOI:10.1109/7260.928927

[12] H. Hsu, M. J. Hill, R. W. Ziolkowski and J. Papapolymerou: IEEE Antennas Wireless Propag. Lett. 1 (2002) 67. DOI:10.1109/LAWP.2002.802548

[13] Y. Guo, S. Kim, X. Liu, H. Gao and G. P. Li: IEEE Trans. Compon. Packag. Manuf. Technol. 5 (2015) 668. DOI:10.1109/TCPMT.2015.2423294

\section{Introduction}

WiMax has attracted much attention for long-range wireless network, especially for broadband wireless access in 2.0-11.0 GHz supported by IEEE802.16 standard. A compact bandpass filter capable of being configured to work at any frequency in the band is one of important components in multiband operating WiMax systems. It facilitates implementation of configurable front ends to miniaturize the systems.

Different kinds of bandpass filters have been proposed and studied intensively to work in a large working frequency range. For planar microstrip filters, working frequency range of $4.14-6.26 \mathrm{GHz}$ [1] and $1.178-3.60 \mathrm{GHz}$ [2] have been demonstrated by filters with footprints of about $17 \times 20 \mathrm{~mm}^{2}$ and $13 \times 18 \mathrm{~mm}^{2}$ respectively. However, due to the utilization of quarter wavelength resonators, planar microstrip filter is unable to cover large frequency range. In contrast, capacitivepost loaded evanescent mode cavities are demonstrated capable of covering wider working frequency of $1.90-5.00 \mathrm{GHz}$ [3] and $0.98-3.48 \mathrm{GHz}$ [4] by sizes of $30.0 \times 18.0 \times 4.5 \mathrm{~mm}^{3}$ and $41.5 \times 24.9 \times 3.17 \mathrm{~mm}^{3}$. Furthermore, when microfabricated in Silicon, such a filter design shows a size of $10.0 \times 5.0 \times 2.5 \mathrm{~mm}^{3}$ and working frequency of $6.0-24.0 \mathrm{GHz}$ [5]. However, its return loss (S11) and insertion loss (S21) are not satisfactory due to limitation of relatively narrow band energy coupling capability of the cavity design.

Electromagnetic Bandgap (EBG) filter has also emerged as an alternative design attributed to its high unloaded quality factor $(\mathrm{Qu})$, ease of integration and low cost $[6,7,8,9,10,11,12]$. However, the proposed EBG filters/resonators are implemented by modification of periodic lattice in EBG substrates which limits its use for broadband coverage.

Instead of relying on modification of a periodic lattice in EBG substrate, a CEBG (Configurable EBG) filter with surface mounted capacitors and broadband energy coupling design is proposed in [13]. The filter is fabricated by common two layer PCB process on FR4 substrate and corresponding periodic lattice in EBG substrate is made up of hollow copper posts. Working frequency of the filter is 
between measurement and simulation results is demonstrated, the filter [13] is only capable of working up to $4.07 \mathrm{GHz}$ due to relatively big insertion loss (IL) at higher frequency. It is still lacking for implementation of a filter with both broadband coverage and acceptable insertion loss and return loss (RL).

In this paper, a novel design of an EBG based bandpass filter is presented to achieve targeted performance while alleviating the constraint imposed by aforementioned insertion loss issue. To implement a filter with both broadband coverage and acceptable IL, RL on demand, equivalent circuit model of an EBG based bandpass filter is proposed and analyzed. High frequency Rogers RO4350 substrate with relatively low dielectric tolerance and loss is used to facilitate broadband working of the filter. The presented bandpass filter is demonstrated to be capable of operating in a broadband working frequency range with customizable selected center frequency.

\section{Filter design and analysis}

Shown in Fig. 1(a) is a customizable EBG bandpass filter to operate at broadband frequency range. This two-pole EBG filter realized by two symmetrical resonators has resin filled copper posts placed periodically in a rectangular lattice. The small spacing between those posts contributes to an effective energy confinement. Energy coupling between adjacent resonators is affected by via post's diameter. Internal energy coupling coefficient and bandwidth of the filter are capable to be adjusted by changing the diameter.

To couple signal energy into the EBG frame made up of copper posts, an energy transition and coupling circuit is designed on the top plate as depicted in Fig. 1(b). The circuit is made up of Co-Planar Waveguide (CPW) line and rectangular slots. Signals go through CPW input port in CPW mode and are transformed into slotline mode coupled to the EBG frame by rectangular slots. Rectangular slots are used instead of common slotlines to increase inductive reactance and corresponding magnetic energy stored at the end of the slots. Consequently, external energy coupling to the filter is enhanced leading to a lower insertion loss of the filter. Moreover, broadband energy transition and coupling is achieved by the rectangular open slots.

Shown in Fig. 1(c) is the design of the bottom plate for customizable adjusting the operating frequency. Lumped capacitors are surface mounted between circular metal pads and bottom plate. Since the working frequency of the filter depends strongly on the value of the capacitors and weakly on the EBG substrate, a broadband operating frequency can be achieved by this compact filter design. Cross section view of the filter is shown in Fig. 1(d). The lumped capacitors are vertically connected with copper posts through shared metal pads.

Equivalent circuit of the EBG based bandpass filter is shown in Fig. 2. Transformers and $\pi$ network represent external and inner energy coupling of the filter respectively. For each resonator, its $\mathrm{LC}$ resonance is determined by $\mathrm{L}_{\text {post }}, \mathrm{C}_{\text {lumped }}$ $\left(\mathrm{C}_{L}\right)$ and $\mathrm{C}_{\text {original }}\left(\mathrm{C}_{\text {orig }}\right)$. $\mathrm{L}_{\text {post }}$ is inductance of the copper post which is vertically connected with a lumped capacitor through shared circular metal pad. $\mathrm{C}_{\text {lumped }}$ is capacitance value of lumped capacitor and it is capable to be adjusted for a 


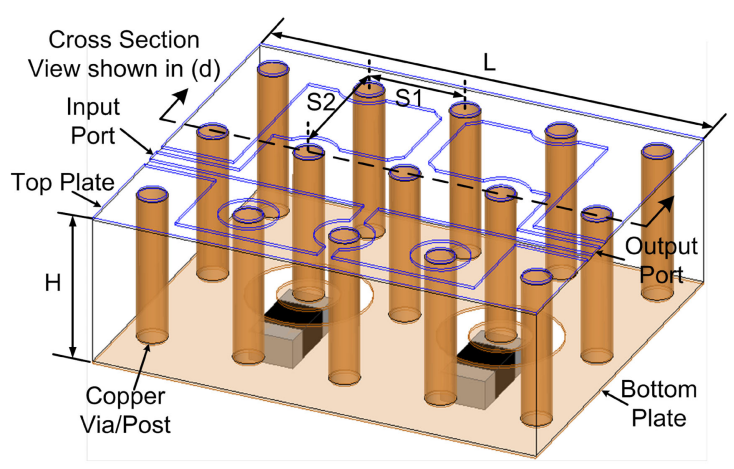

(a)

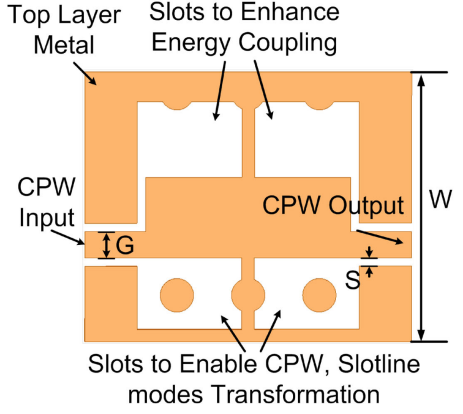

(b)

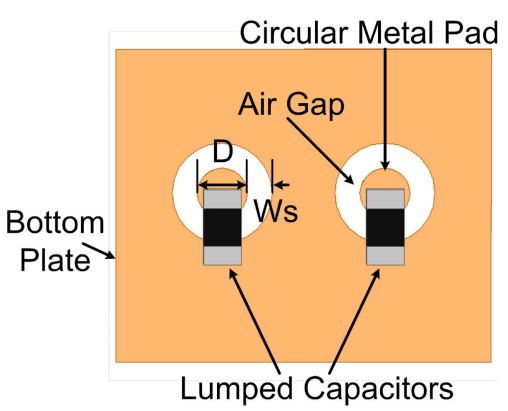

(c)

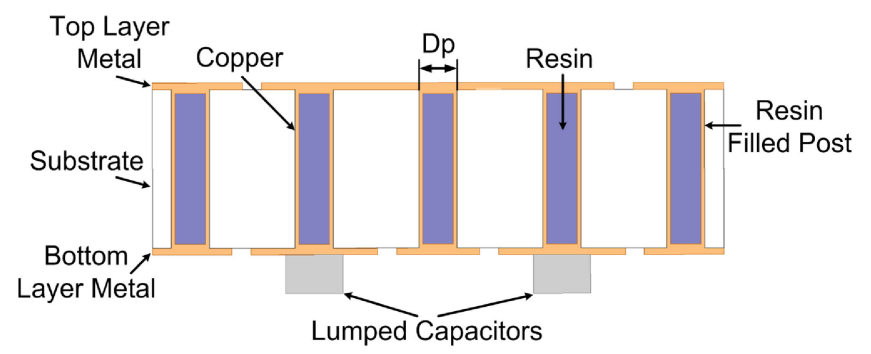

(d)

Fig. 1. Schematic of presented filter design $(\mathrm{L}=3.00, \mathrm{~W}=2.50$, $\mathrm{H}=1.00, \quad \mathrm{Dp}=0.20, \quad \mathrm{~S} 1=0.65, \quad \mathrm{~S} 2=0.90, \quad \mathrm{G}=0.31$, $\mathrm{S}=0.10, \mathrm{Ws}=0.10, \mathrm{D}=0.36$, all in millimeters) (a) $3 \mathrm{D}$ diagram of the filter with generalized lumped capacitors as loading elements (b) Top view of the filter (c) Bottom view of the filter (d) Cross section view.

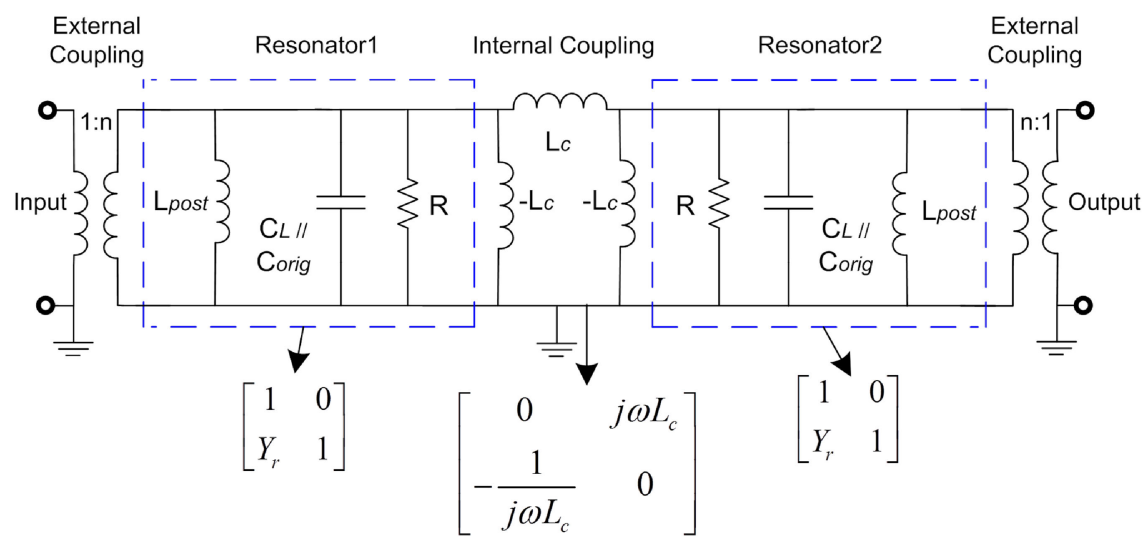

Fig. 2. Equivalent circuit of the EBG based bandpass filter. 
specified working frequency of the filter. $C_{\text {orig }}$ stands for the original capacitance of EBG substrate which is affected by thickness and dielectric constant of substrate. $\mathrm{R}$ represents energy loss of each resonator.

The filter's characteristic of S21 is analyzed based on the equivalent model. ABCD matrixs of three sections of the model are shown in Fig. 2.

$$
\text { Where } Y_{r}=j \omega C_{L}+j \omega C_{\text {orig }}+\frac{1}{j \omega * L_{\text {post }}}+\frac{1}{R}
$$

The total ABCD matrix of these three cascade sections is:

$$
\left[\begin{array}{ll}
A & B \\
C & D
\end{array}\right]=\left[\begin{array}{cc}
j \omega L_{c} Y_{r} & j \omega L_{c} \\
-\frac{1}{j \omega L_{c}}+j \omega L_{c} Y_{r}^{2} & j \omega L_{c} Y_{r}
\end{array}\right]
$$

Using matrix transformation equation, S21 of these three cascade sections can be calculated by:

$$
\begin{aligned}
S_{21} & =\frac{2}{A+\frac{B}{Z_{1}}+C Z_{1}+D}=\frac{2}{j 2 \omega L_{c} Y_{r}+\frac{j \omega L_{c}}{Z_{1}}-\frac{Z_{1}}{j \omega L_{c}}+j Z_{1} \omega L_{c} Y_{r}^{2}} \\
& \approx \frac{2}{\frac{2 L_{c}}{L_{\text {post }}}+\frac{2 Z_{1} L_{c}}{R L_{\text {post }}}-\frac{2 Z_{1} \omega^{2} L_{c}\left(C_{\text {orig }}+C_{L}\right)}{R}+j\left(\frac{2 \omega L_{c}}{R}+\frac{Z_{1}}{\omega L_{c}}+\frac{Z_{1} \omega L_{c}}{R^{2}}-\frac{Z_{1} L_{c}}{\omega L_{\text {post }}^{2}}\right)}
\end{aligned}
$$

From this equation, insertion loss of the filter is directly related with $\mathrm{R}$ of each resonator. With other parameters the same, a lower insertion loss will be achieved by increasing the value of $R$.

$\mathrm{Q} u$ (Unloaded Quality Factor) of resonator is given by [12]:

$$
Q_{u}=\left(\frac{1}{Q_{\text {conductor }}}+\frac{1}{Q_{\text {dielectric }}}\right)^{-1}
$$

Where $\frac{1}{Q_{\text {dielectric }}}=\tan \delta$ which is loss tangent of filter's substrate material. From the paralleled LC resonance network shown in Fig. 2, Qu of resonator is also equal to:

$$
Q_{u}=\omega_{0}\left(C_{L}+C_{\text {orig }}\right) R
$$

By substituting Eq. (5) into Eq. (4), the analytical expression of $R$ with respect to $\tan \delta$ can then be found.

$$
R=\frac{Q_{\text {conductor }}}{\omega_{0}\left(1+\tan \delta Q_{\text {conductor }}\right)\left(C_{L}+C_{\text {orig }}\right)}
$$

With other parameters the same, $\mathrm{R}$ will be increased by reducing $\tan \delta$. Considering $\tan \delta$ is substrate material's loss tangent, lower insertion loss will be achieved by the filter when high frequency low loss substrate is used.

\section{Experimental results and discussions}

Full-wave simulation solver HFSS is used in theoretic design of the bandpass filter. Rogers RO4350 substrate with thickness of $1.00 \mathrm{~mm}$, dielectric constant of 3.66 and loss tangent of 0.004 is used for implementation of the filter due to its low dielectric tolerance and loss at high frequency. In implementing the filter, a two 
layer metal PCB process is used to construct the metal via posts, top and bottom metal plates.

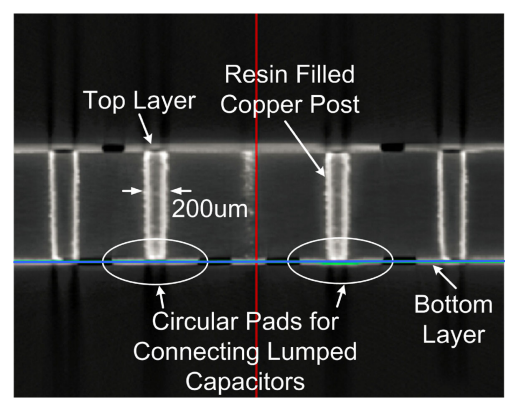

Fig. 3. Nano CT image of cross section view of filter implemented on RO4350 substrate.

The fabricated EBG substrate is scanned by Nano CT Xradia 410 and corresponding cross section view of the EBG substrate is shown in Fig. 3. As what is shown in the figure, resin filled solid copper posts are connecting the top copper layer with bottom layer. Solid circular pads are designed for a good connection with lumped capacitors by manually soldering.

Fig. 4 shows photomicrographs of the fabricated filter. The fabricated filter is measured using an HP 8510C network analyzer and Cascade Microtech groundsignal-ground probes with a 200 -um pitch size. A short-open-load-through is adopted for calibration of measurements.

ATC600L series lumped capacitors are chosen for their small size and relatively high Q factor in implementation of the customizable EBG bandpass filter. Bottom view of the filter loaded by capacitors is shown in Fig. 4(b). Working frequency of the bandpass filter is easily adjusted by loading different value capacitors on the filter. Measurement results of filter are shown in Fig. 4(c). Those results are in good agreement with simulation results. The filter's center frequency increases from 2.6 GHz up to $10.0 \mathrm{GHz}$ with acceptable insertion loss, return loss as the surface mounted lumped capacitors' value reduces from $5.1 \mathrm{pF}$ to $0.3 \mathrm{pF}$.

Insertion loss and corresponding $\mathrm{Q} u$ of the filter depend on the lumped capacitors' $\mathrm{Q}$ factor which is affected by its value and filter's working frequency [13].

As shown in Fig. 4(c), when the filter is loaded by different capacitor and working at different frequency, insertion loss of the filter changes from $3.34 \mathrm{~dB}$ to $1.20 \mathrm{~dB}$ as corresponding surface mounted capacitors' $\mathrm{Q}$ factor increase from 89 to 172.4. The corresponding measured return loss changes from $20.7 \mathrm{~dB}$ to $9.6 \mathrm{~dB}$. The $\mathrm{Q} u$ of the filter is analyzed, suggesting that it is limited by the $\mathrm{Q}$ of surface mounted lumped capacitors and not by the EBG substrate.

Center working frequency of the filter can be estimated by using equation shown in Fig. 5. In this equation, $\mathrm{C}_{\text {lumped }}$ is capacitance value of surface mounted lumped capacitors. $\mathrm{L}_{\text {post }}$ is inductance of copper post which is connected with the lumped capacitor. $C_{\text {orig }}$ stands for the original capacitance of EBG substrate. Based on this equation, $\omega^{2}$ vs $1 / C_{\text {lumped }}$ curve is extracted from test results and shown in 


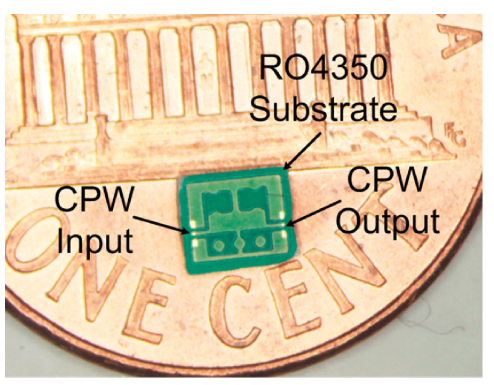

(a)

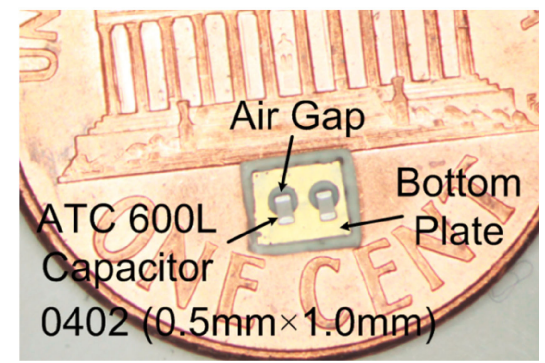

(b)
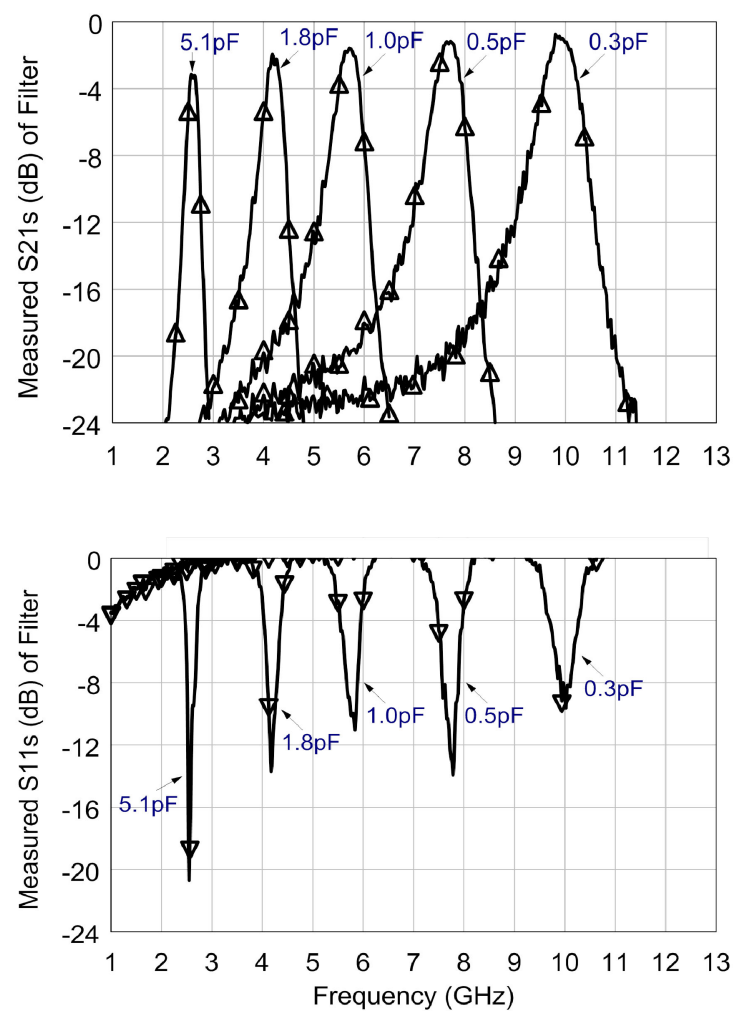

(c)

Fig. 4. Photomicrographs and measurement results of fabricated customizable EBG bandpass filter in RO4350 substrate (a) Top view of proposed filter (b) Bottom view of filter (c) Measurement results of the filter loaded with different value lumped capacitors.

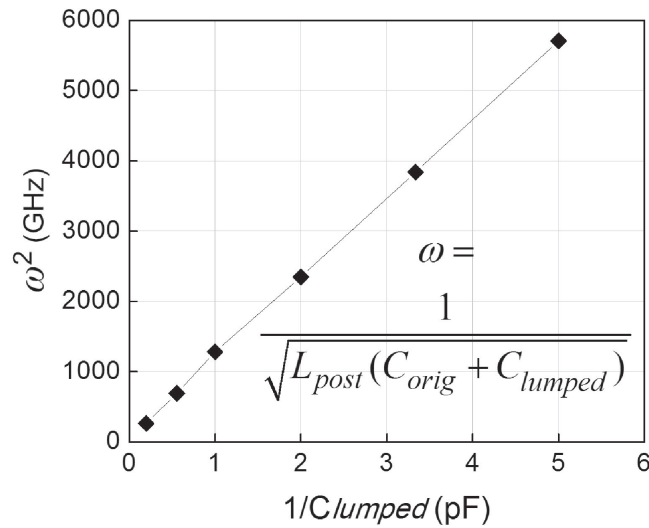

Fig. 5. Measured filter's $\omega^{2}$ when surface mounted by different lumped capacitors $\left(\mathrm{C}_{\text {lumped }}\right)$. 
$L_{\text {post }}$ is determined by magnetic energy storage capability of EBG substrate and controlled by diameter of post and thickness of the substrate. From Fig. 5, it is demonstrated that $L_{\text {post }}$ is almost constant from 2.6 to $10.0 \mathrm{GHz}$ which imply that the filter is capable of working at any specified frequency within the range by customizable selecting lumped capacitors.

\section{Conclusion}

In this letter, a novel customizable EBG filter is proposed with a compact size of $2.5 \times 3.0 \times 1.0 \mathrm{~mm}^{3}$ and operating from 2.6 to $10.0 \mathrm{GHz}$. The bandpass filter is demonstrated using PCB manufacturing technology. The measured results confirm that acceptable insertion loss and return loss in the entire working frequency range is achievable for the filter and that it is capable of working at specified frequency by customizable selecting lumped capacitors. The current design of the filter suggests that a compact tunable filter operating at broadband is achievable, when the lumped capacitors are replaced by varactors or PIN diodes.

\section{Acknowledgments}

This work is supported by the national natural science foundation of China, (No. 11274166, No. 11474160). 